\title{
Deficiency of pigment epithelium-derived factor in nasopharyngeal carcinoma cells triggers the epithelial-mesenchymal transition and metastasis
}

\author{
Ting Zhang ${ }^{1,2,3,9}$, Ping Yin ${ }^{2,9}$, Zichen Zhang ${ }^{4,9}$, Banglao Xu ${ }^{3}$, Di Che ${ }^{1,2}$, Zhiyu Dai ${ }^{2}$, Chang Dong ${ }^{2}$, Ping Jiang ${ }^{2}$, Honghai Hong ${ }^{2,5}$, \\ Zhonghan Yang ${ }^{2}$, Ti Zhoü ${ }^{2}$, Jianyong Shao ${ }^{*, 4}$, Zumin Xu ${ }^{\star, 2,6}$, Xia Yang ${ }^{*, 1,2,7}$ and Guoquan Gao ${ }^{*, 1,2,8}$
}

Distant metastasis is the primary cause of nasopharyngeal carcinoma (NPC) treatment failure while epithelial-mesenchymal transition (EMT) is the critical process of NPC invasion and metastasis. However, tumor-suppressor genes involved in the EMT and metastasis of NPC have not been explored clearly compared with the oncogenes. In the present study, the expression of pigment epithelium-derived factor (PEDF), a potent endogenous antitumor factor, was diminished in human NPC tissues and associated with clinicopathological and EMT features. The knockdown of PEDF induced EMT in lower metastatic NPC cell lines and overexpression of PEDF restored epithelial phenotype in higher metastatic NPC cell lines with typical EMT. The inhibition of PEDF mediated NPC cell spontaneous metastasis in vivo. LRP6/GSK3 $\beta / \beta$-catenin signal pathway rather than AKT/GSK3 $\beta$ pathway was involved in the effects of PEDF on EMT. The expression of PEDF was directly downregulated by elevated miR-320c in NPC. In conclusion, our findings indicate for the first time that PEDF functions as tumor-suppressor gene in the occurrence of EMT and metastasis in NPC. PEDF could serve as a promising candidate for NPC diagnosis, prognosis and treatment.

Cell Death and Disease (2017) 8, e2838; doi:10.1038/cddis.2017.114; published online 1 June 2017

Nasopharyngeal carcinoma (NPC) is prevalent in southern China and South-East Asia, with an annual incidence rate of about 25-30 per 100000 people, wheras it is rare in the Western world (1 per 100000$).{ }^{1}$ Although NPC is sensitive to radiotherapy and chemotherapy, the overall 5-year survival rate of NPC patients is around $80 \%, 20-30 \%$ patients develop distant metastasis or loco-regional recurrence eventually leading to death. ${ }^{2}$ Therefore, understanding the mechanism of NPC metastasis and identification of effective anti-nasopharyngeal carcinoma metastasis drugs have been emerging as a promising direction for the treatment of NPC.

Recent studies have shown that epithelial-mesenchymal transition (EMT) is closely related to tumor metastasis. EMT occurs in embryogenesis, fibrosis and invasion of the tumor. They have many common characteristics, such as the loss of the connection between epithelial cells and epithelial cell phenotypic markers, increases mesenchymal markers and endows cell migration ability. ${ }^{3}$ EMT is closely related to the invasion and metastasis of NPC as well, inhibition of NPC cell's EMT could significantly suppress the metastasis of NPC. ${ }^{4-7}$
The canonical Wnt/ $\beta$-catenin pathway is involved in various biological processes, including embryonic development, stem cell maintenance and tumorigenesis. When $\mathrm{Wnt} / \beta$-catenin pathway was activated, the core protein $\beta$-catenin translocates into cell nucleus and directly involve in gene transcription and cell adhesion. ${ }^{8}$ Zeng et al. $^{9}$ and co-workers observed Wnt pathway was abnormally activated in NPC using nasopharyngeal tissue array. It has been reported that activation of Wnt/ $\beta$-catenin pathway by oncogenes could promote EMT and metastasis in NPC. ${ }^{10-12}$ However, tumor-suppressor genes involved in the EMT and metastasis of NPC have not been identified.

Pigment epithelium-derived factor (PEDF) is a potent and versatile endogenous inhibitor of angiogenesis. ${ }^{13}$ Previous studies demonstrated that PEDF is a favorable prognostic indicator in colorectal, pancreatic, lung and breast cancer. ${ }^{14-17}$ There is a complex mechanism underlying the antitumor effects of PEDF, which includes inhibition of angiogenesis and tumor cell migration, induction of apoptosis and pro-tumor differentiation in certain tumor cell types. ${ }^{18}$ However, it is still unclear the exact role of endogenous PEDF in EMT occurrence and NPC metastasis. Previously, we have reported that PEDF could bind

\footnotetext{
${ }^{1}$ Program of Molecular Medicine, Affiliated Guangzhou Women and Children's Hospital, Zhongshan School of Medicine, Sun Yat-sen University, Guangzhou 510080, China; ${ }^{2}$ Department of Biochemistry, Zhongshan School of Medicine, Sun Yat-sen University, Guangzhou 510080, China; ${ }^{3}$ Department of Laboratory Medicine, Guangzhou First People's Hospital, Guangzhou Medical University, Guangzhou 510180, China; ${ }^{4}$ Department of Molecular Diagnostics, Sun Yat-sen University Cancer Center, Guangzhou 510160, China; ${ }^{5}$ Department of Clinical Laboratory, Third Affiliated Hospital of Guangzhou Medical University, Guangzhou 510150, China; ${ }^{6}$ Cancer Center, Affiliated Hospital of Guangdong Medical College, Zhanjiang 524001, China; ${ }^{7}$ Guangdong Engineering \& Technology Research Center for Gene Manipulation and Biomacromolecular Products (Sun Yat-sen University), Guangzhou 510080, China and ${ }^{8}$ China Key Laboratory of Tropical Disease Control (Sun Yat-sen University), Ministry of Education, Guangzhou 510080, China

*Corresponding author: J Shao, Z Xu, X Yang or G Gao, Program of Molecular Medicine, Affiliated Guangzhou Women and Children's Hospital, Zhongshan School of Medicine, Sun Yat-sen University, 74 Zhongshan 2nd Road, Guangzhou 510080, China. Tel: +86-20-87332128; Fax: +86-20-87332128; E-mail: shaojy@ sysucc.org.cn, zuminxu@163.com, yangxia@mail.sysu.edu.cn or gaogq@mail.sysu.edu.cn

${ }^{9}$ These authors contributed equally to this work.

Received 31.12.16; revised 18.2.17; accepted 20.2.17; Edited by A Stephanou
} 
to low-density lipoprotein receptor-related protein 6 (LRP6) and hinder the Wnt ligand-induced dimerization of LRP6 and Fz receptor, thus block the activation of canonical Wnt signaling in diabetic complications. ${ }^{19,20}$ It is unknown whether PEDF could also block Wnt/ $\beta$-catenin activation and further suppress EMT and metastasis in NPC.

From the clinical tissue chips analysis, we found the extremely low expression of PEDF in NPC was closely associated with advanced clinicopathological stage. Therefore, the hypothesis of PEDF as a possible tumor-suppressor gene and the critical role of PEDF deficiency in the occurrence of EMT and metastasis in nasopharyngeal carcinoma cells will be explored. Further, the underlying mechanism of PEDF on EMT and the expression regulation of PEDF will also be investigated in the present study.

\section{Results}

PEDF expression is diminished in human NPC cells, associated with clinicopathological and EMT features. To determine whether PEDF expression was modulated during nasopharyngeal carcinoma progression, we measured PEDF expression in the nasopharynx epithelial tissues (NET) and NPC tissues (Figure 1a). The clinical characteristics of 218 NPC patients were presented in Supplementary Table 3. All (12/12) of NET showed strong positive staining, but only $7.3 \%(16 / 218)$ of NPC tumor tissues had weak staining (Figure 1b). Next, the tumors were categorized as positive or negative for PEDF. We observed NPC patients of negatively PEDF expression had advanced pathological tumor stage and clinical stage. No significant correlation between PEDF expression and pathological node stage was observed (Table 1). EMT is the critical process of NPC invasion and metastasis. To explore the relationship between PEDF and EMT, we performed immunohistochemical staining of PEDF, E-cadherin and vimentin in another 12 NET samples and 20 NPC biopsies. As shown in Figures 1c-e, PEDF expression level positively correlated with the expression level of E-cadherin $(r=0.427, P<0.05)$, and inversely related to the expression level of vimentin $(r=-0.414$, $P<0.05)$. Next, real-time PCR and western blot was performed to quantify the levels of PEDF among a series of NPC cells. Results showed low-metastatic CNE-2 and SUNE-1 cells had high PEDF expression, which was accompanied by reduced levels of mesenchymal markers vimentin and $\mathrm{N}$-cadherin and elevated levels of epithelial proteins E-cadherin and $a$-catenin compared with high-metastatic S18 and 5-8F cells (Figures if and $\mathrm{g}$ ). All these data suggested that PEDF might have an inhibitory effect on EMT.

Knockdown of endogenous PEDF expression in NPC cells induces EMT. To examine the causal role of PEDF in EMT of NPC cells, we engineered cell lines from lowmetastatic CNE-2 and SUNE-1 cells that stably expressed either shRNA targeting PEDF expression (shPEDF) or a scrambled nontarget shRNA. As shown in Figure 2a, knockdown of PEDF led to the transformation of the cobblestonelike cells CNE-2 and SUNE-1 to spindle-like, fibroblastic cells. Furthermore, in 3D culture, PEDF-knockdowned CNE-2 and SUNE-1 cells grew into more structurally well-organized spheres with invasive projections emanating from the cells when compared with the scrambled control cells (Figure $2 b$ ). We also observed that the invasive and migratory properties were enhanced upon silencing PEDF (Figure 2c). Also, the typical EMT markers were changed includes the downregulation of epithelial markers E-cadherin and $a$-catenin, upregulation of mesenchymal markers vimentin and $\mathrm{N}$-cadherin (Figure 2d). These results proved that knockdown of PEDF is sufficient to induce EMT in NPC cells.

Overexpression of PEDF induces restoration of the epithelial phenotype. To further investigate the impact of PEDF on EMT in NPC cells, we overexpress PEDF in the high-metastatic S18 and 5-8 F cells. As shown in Figure 3a, S18 and 5-8F cells were transformed from spindle-lie and fibroblastic phenotype to cobblestone-like after overexpressing PEDF. In 3D culture, S18/Vector and 5-8 F/Nector cells grew into more structurally well-organized spheres with invasive projections emanating from the cells when compared with the S18/PEDF and 5-8 F/PEDF cells (Figure 3b). The migration and invasion abilities of S18 and 5-8 F cells were also reduced in PEDF-overexpressing cells (Figure $3 \mathrm{c}$ ). Western blot confirmed that reduced expression of the epithelial markers, increased expression of the mesenchymal markers upon PEDF overexpression (Figure 3d). These results confirmed that PEDF could inhibit NPC cells migration and invasion through suppression of EMT.

Knockdown of PEDF mediates the distant metastasis in vivo. As the low PEDF expression was associated with NPC metastatic traits, we next asked whether PEDF could mediate NPC cells metastasis in vivo. The effect of PEDF in vivo was determined by using a spontaneous spleen transfer to liver model. We found mice bearing CNE-2/ shPEDF and S18/Vector primary tumors displayed more invasive phenotype, which included the NPC cells invaded into the normal spleen tissues and formed small metastasis, compared with nude mice injected with CNE-2/scramble or S18/PEDF cells (Figure 4a). As expected, PEDF knockdown cells showed a significant increased liver metastasis and weight, and the liver metastasis was dramatically inhibited as well as the weight of liver was decreased by overexpressing PEDF (Figures $4 b-d$ ). All of these data confirmed that PEDF has a pivotal role in NPC metastasis in vivo.

Wnt/ $\beta$-catenin signaling pathway is required for PEDFmediated EMT. We have previously reported that PEDF serves as a novel inhibitor of the canonical Wnt pathway, binds to Wnt co-receptor low-density lipoprotein receptorrelated protein 6 (LRP6) and blocks the signaling by antagonizing Wnt ligands in retinal pigment epithelial cells. $^{19}$ A higher level of nuclear $\beta$-catenin was found in high-metastatic S18 and 5-8 F cells compared with lowmetastatic CNE-2 and SUNE-1 cells (Supplementary Figures $1 \mathrm{~A}$ and $\mathrm{B})$. Consequently, we validate whether $\mathrm{Wnt} / \beta$-catenin signaling pathway is responsible for the inhibitory effect of PEDF in NPC. To further confirm a direct modulation of Wnt/ $\beta$-catenin by PEDF, we evaluate the effect of PEDF on the nuclear translocation of $\beta$-catenin. PEDF could decrease the elevated nuclear $\beta$-catenin level in S18 and 5-8 F cells 
(Figure 5a). The subcellular fractionation results also confirmed that PEDF could decrease the nuclear $\beta$-catenin levels (Figure $5 b$ ). Strikingly, the inhibited EMT and migration in NPC cells caused by overexpressing PEDF were restored by $\mathrm{LiCl}$, which was used to activate $\beta$-catenin (Figures $5 \mathrm{c}$ and d). Next, we used Wnt3a, the canonical Wnt/ $\beta$-catenin a
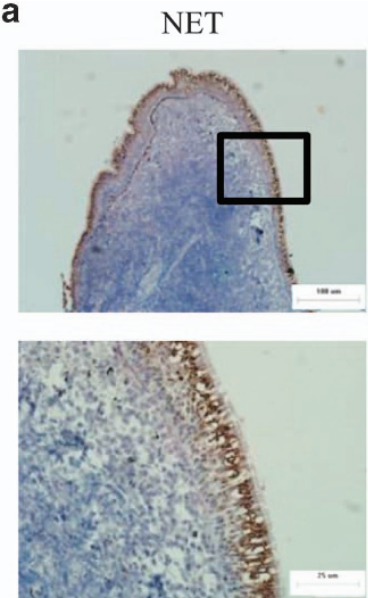

C
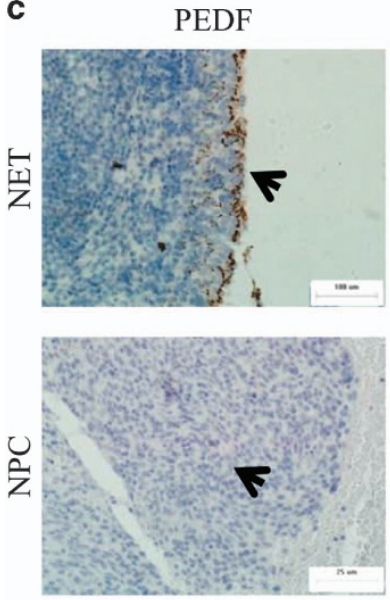

f

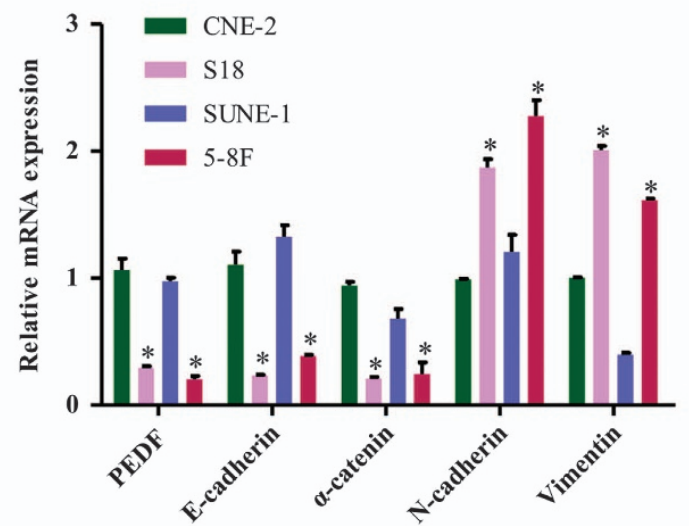

NPC 1
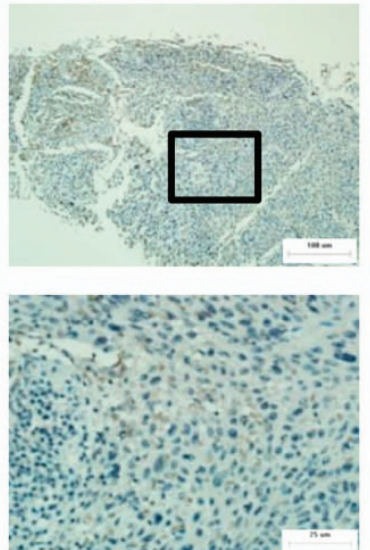

E-cadherin
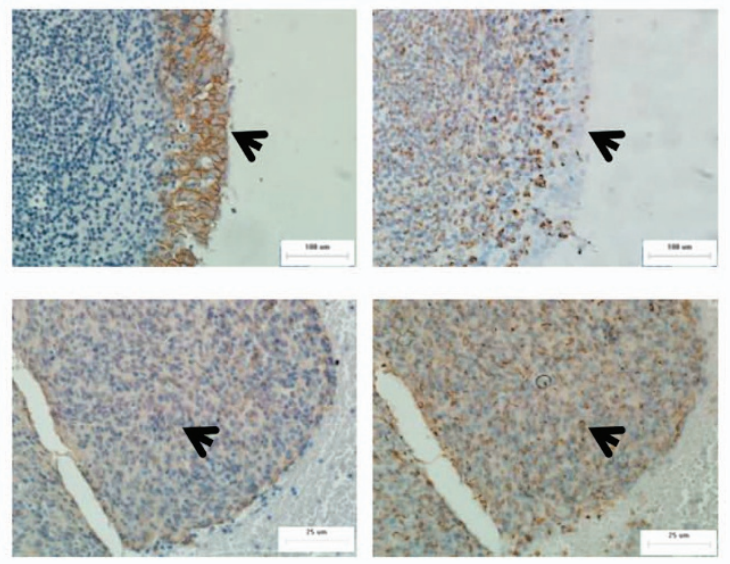

NPC 2
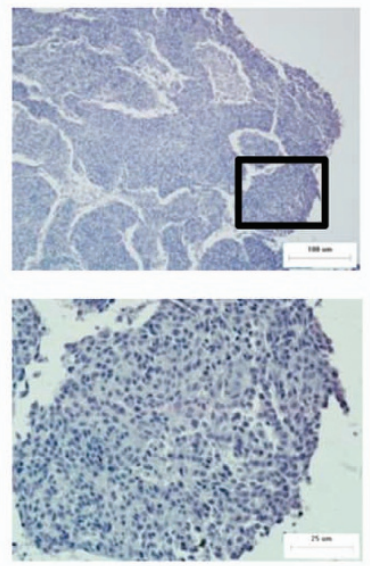

Vimentin

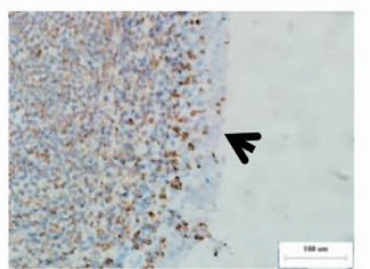

d

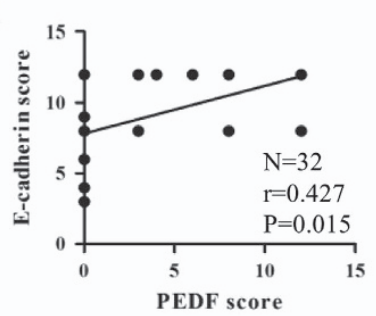

e
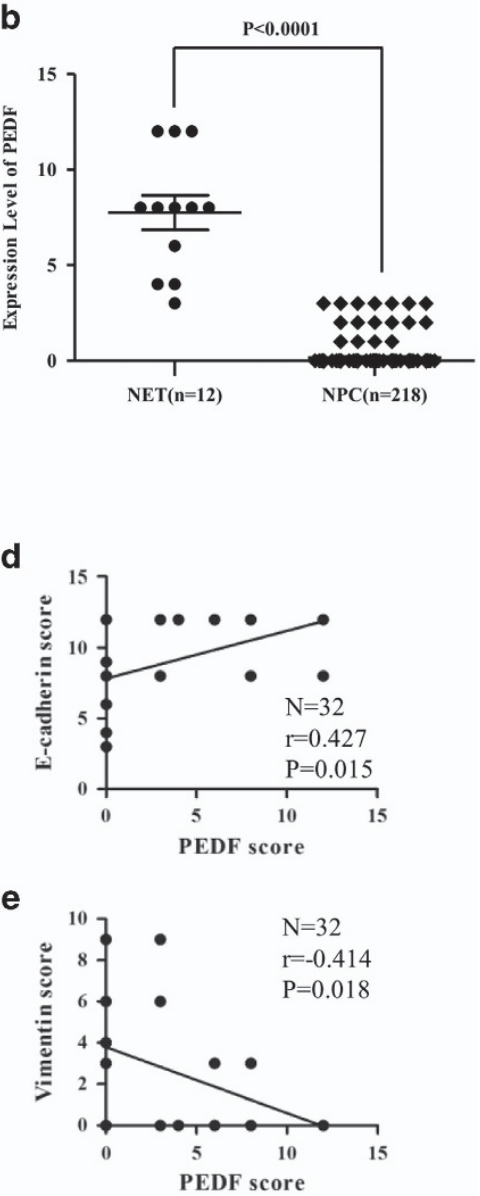

g

CNE-2 S18 SUNE-1 5-8F

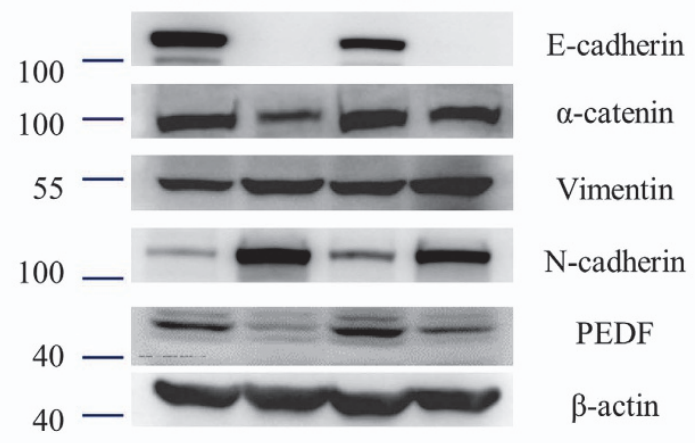

Figure 1 Pigment epithelium-derived factor (PEDF) expression is diminished in human nasopharyngeal carcinoma (NPC) cells, associated with clinicopathological and epithelial-mesenchymal transition (EMT) features. (a) The representative area of nasopharynx epithelial tissues (NET) or NPC. NPC 1, weak PEDF expression. NPC 2, negative PEDF expression. (b) Quantitative analysis of staining intensity for PEDF in various tissues. (c) The expression of PEDF, E-cadherin and Vimentin in a cohort of NET biopsy samples and NPC samples using immunohistochemistry assay. (d and e) PEDF significantly correlates with E-cadherin and Vimentin in a cohort of NET biopsy samples and NPC samples. (f and $\mathbf{g}$ ) Real-time PCR and western blot (WB) analysis of the expression levels of PEDF in a series of human NPC cell lines, normalized to $\beta$-actin levels. Bars correspond to mean \pm standard deviation (S.D.), ${ }^{*} P<0.05$ 
Table 1 Correlation between PEDF and clinicopathologic characteristics of patients with NPC

\begin{tabular}{lrrrr}
\hline & & \multicolumn{2}{c}{ PEDF expression level } & \\
\cline { 3 - 3 } Characteristic & Number & Positive & Negative & P-value \\
\hline T stage & & & & $\mathbf{0 . 0 1 8}$ \\
T1-2 & 99 & $12(12.1 \%)$ & $87(87.9 \%)$ & \\
T3-4 & 119 & $4(3.3 \%)$ & $115(96.7 \%)$ & \\
N stage & & & & \multirow{2}{*}{0.292} \\
N0-1 & 132 & $12(9.1 \%)$ & $120(90.9 \%)$ & \\
N2-3 & 86 & $4(4.7 \%)$ & $82(95.3 \%)$ & \\
Clinical staging & & & & $\mathbf{0 . 0 4 5}$ \\
I-II & 68 & $9(13.2 \%)$ & $59(86.8 \%)$ & \\
III-IV & 150 & $7(4.7 \%)$ & $143(95.3 \%)$ & \\
\hline
\end{tabular}

$P<0.05$ were presented in bold and italic

pathway ligand, to activate the $\mathrm{Wnt} / \beta$-catenin pathway and found that Wnt3a could increase the total Wnt co-receptor LRP6 level and decrease the expression of E-cadherin thus promote the cell migration, while these functions could be reversed by PEDF. Furthermore, PEDF-inhibited Wnt3a activation was abolished by $\mathrm{Wnt} / \beta$-catenin signaling intracellular agonist $\mathrm{LiCl}$ (Figures $5 \mathrm{e}$ and $\mathrm{f}$ ), which suggests that PEDF regulates $W n t$ signaling at the receptor level. These results showed that inhibition of $W n t / \beta$-catenin signaling pathway is required for PEDF-inhibited EMT in NPC.

PEDF is a direct target of miR-320c in NPC. As PEDF has an important role in NPC progression, the reason for the diminished PEDF observed in NPC is unclear. We have previously found miR-320c was increased in the plasma of NPC patients, ${ }^{21}$ and miRNA target prediction algorithms (RNAhybrid) also identified that there is a potential binding sites of miR-320c in PEDF coding sequence (CDS) region ${ }^{22}$ (Figure 6a). We asked whether miRNA could be responsible for PEDF regulation. The expression level of PEDF and miR-320c was evaluated using qRT-PCR in 11 NET tissues and 27 NPC samples. As shown in Figure 6b, 56\% (14 cases) of samples with low miR-320c expression (25 cases) exhibited high levels of PEDF, whereas $76.9 \%$ (10 cases) of samples with high miR-320c expression (13 cases) showed low expression of PEDF $(P<0.05)$, which indicated that the expression levels of miR-320c were inversely related to the expression levels of PEDF in nasopharynx tissues. Furthermore, western blot and qRT-PCR showed that inhibition of miR-320c increased the endogenous PEDF expression in the NPC cell lines S18 and 5-8 F (Figures $6 \mathrm{c}$ and $\mathrm{d}$ ). Furthermore, reporter assays showed that the activity of luciferase linked with the CDS of PEDF was repressed in miR-320c mimic-transfected 293A cells, compared with those in control cells. Of note, mutations brought into the CDS of PEDF abolished miR-320c suppressive effects (Figures $6 \mathrm{e}$ and f). Collectively, these data suggest that miR-320c directly suppresses PEDF expression by targeting CDS of PEDF.

\section{Discussion}

NPC has the highest rate of invasive and metastasis among head and neck malignant tumor, and distant metastasis is the leading cause of treatment failure. ${ }^{23}$ Therefore, clarifying the underlying mechanism of nasopharyngeal carcinoma metastasis and developing effective targeting drugs became the core issue for the treatment of NPC. The present study provides clinical and experimental evidences to support the tumor-suppressor role of PEDF in NPC. Our results revealed that the deficiency of PEDF is involved in the occurrence of metastasis in NPC patients and confirmed that knockdown of PEDF induces EMT of NPC cells and promotes invasiveness, whereas restoration of PEDF reverses EMT phenotype of NPC. We further demonstrated that PEDF suppress EMT and metastasis depended on its inhibitory effect on $\mathrm{Wnt} / \beta$-catenin pathway. Finally, we verified that the increased miR-320c level in NPC direct downregulates expression of PEDF. Previously, we have reported that PEDF could combine with radiotherapy to enhance the antitumor effects on NPC. ${ }^{24}$ Based on these findings, we believe that PEDF could be an appealing candidate for prevention and treatment of NPC progression.

It has been reported that the distinct oncogenes were activated in the EMT induction of NPC. Epstein-Barr virus induces the initiation, metastasis and recurrence of NPC through its encoded protein LMP1 and LMP2A. ${ }^{5,6} \mathrm{Bmi}-1$ has an important role in the pathogenesis of NPC by inducing EMT via the PI3K/Akt pathway. ${ }^{25}$ However, tumor-suppressor genes involved in the EMT and metastasis of NPC have rarely been identified. To our knowledge, we demonstrate for the first time that PEDF was downregulated in human NPC tissues compared with NET tissues (Figure 1a). Only 7.3\% (16/218) of NPC tumor tissues had PEDF weak expression (Figure 1b). These results suggest that the loss of PEDF might occur in the early stage of NPC. We also observed that NPC patients of negative PEDF expression had advanced pathological tumor stage and clinical stage (Table 1). Furthermore, the loss of PEDF could induce EMT occurrence (Figures 2-4). Taken together, our data provide a biological basis for the feasible role of PEDF as a tumor-suppressor gene in NPC.

In this study, our results identify that PEDF participates in NPC cells' EMT in vitro (Figures 2 and 3). Importantly, this effect was reproduced in vivo (Figure 4 and Supplementary Figure 2). To our knowledge, this is the first study demonstrating an essential role for PEDF in inhibition of EMT. Previous studies showed PEDF is a useful marker for monkey RPE polarization and increased in polarized human RPE cells compared with non-polarized RPE cells while losing apicalbasal polarity is the important step during EMT. ${ }^{18,26,27}$ Orgaz et $a .^{28}$ showed that PEDF expression was correlated with $\mathrm{E}$-cadherin and $\mathrm{N}$-cadherin in some pairing melanoma cells but not SBcl2 cells. Downregulation of PEDF is associated with increased EMT in bladder and breast cancer tiusses. ${ }^{29,30}$ PEDF overexpression in MD-231 breast cancer cells decreased fibronectin and migration but did not change the EMT phenotype in our recent study. ${ }^{31}$ This cell type-specific function has been observed with other proteins, such as Bmi-1 and ILEI. ${ }^{25,32}$ Thus, PEDF-participated EMT may be existed in a cell type-dependent manner. 
a
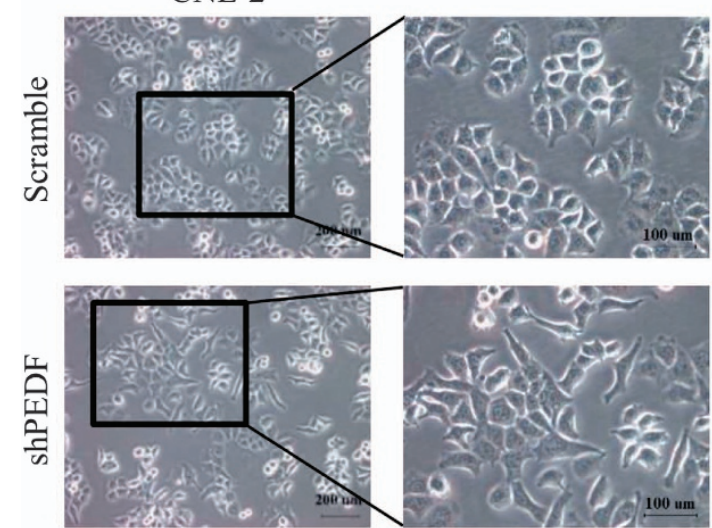

b
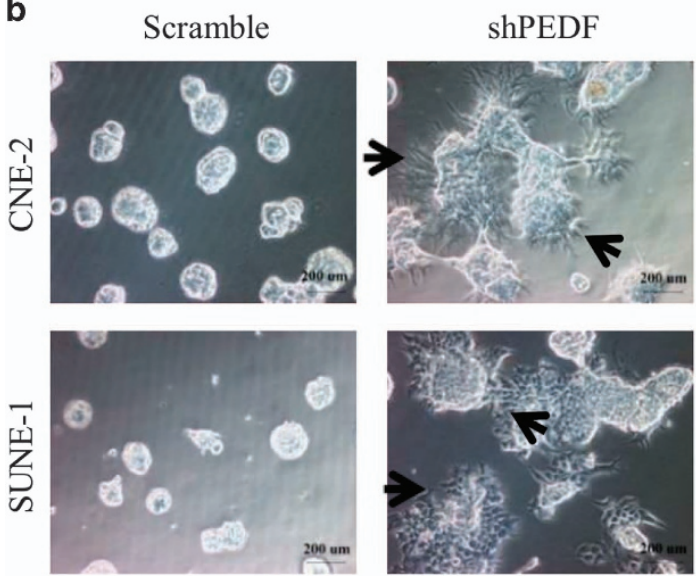

d

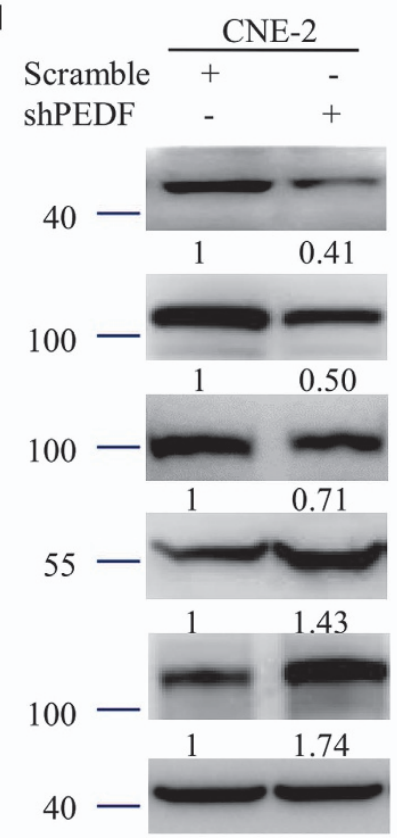

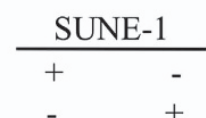

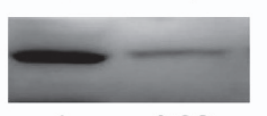

$1 \quad 0.28$

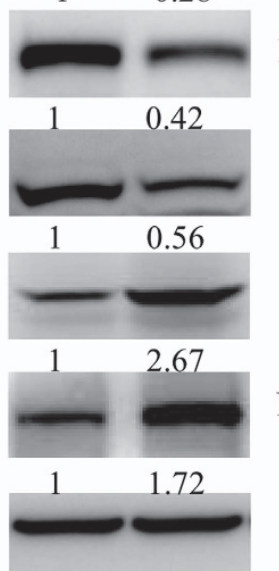

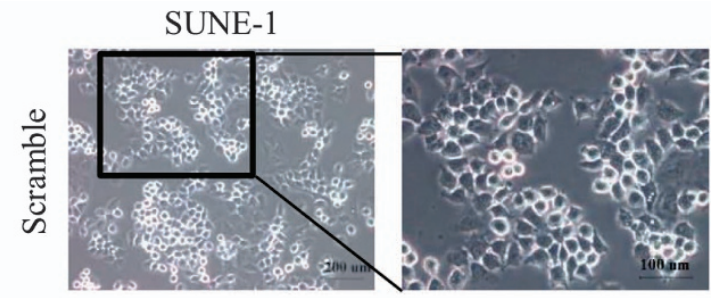

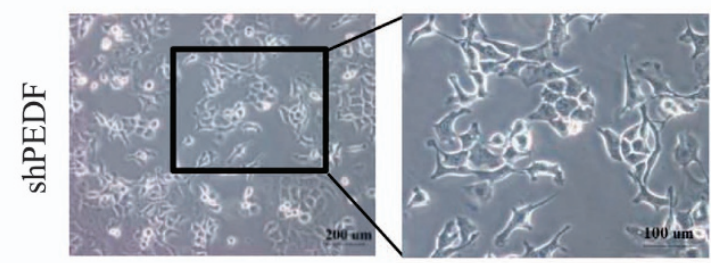

c
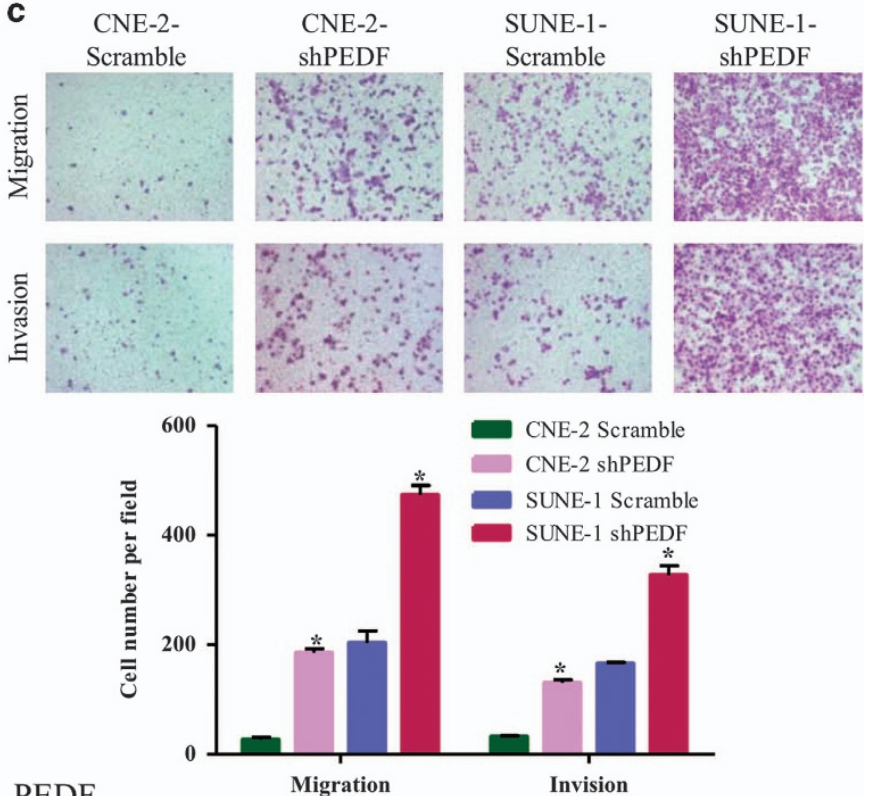

PEDF

E-cadherin

$\alpha$-catenin

Vimentin

$\mathrm{N}$-cadherin

$\beta$-actin

Figure 2 Knockdown of endogenous PEDF expression in NPC cells induces EMT. (a) Cell morphology was evaluated by phase-contrast microscopy. (b) Representative micrographs of indicated cells grown on Matrigel for 8 days in 3D spheroid invasion assay. (c) The migration/invasion ability of indicated cells was evaluated by Transwell assay. (d) Expression of epithelial cell markers (E-cadherin, $\alpha$-catenin) and mesenchymal cell markers (Vimentin, N-cadherin) in indicated cells were examined by WB. $\beta$-actin was used as a loading control. Bars correspond to mean \pm standard deviation (S.D.), ${ }^{*} P<0.05$ 
a
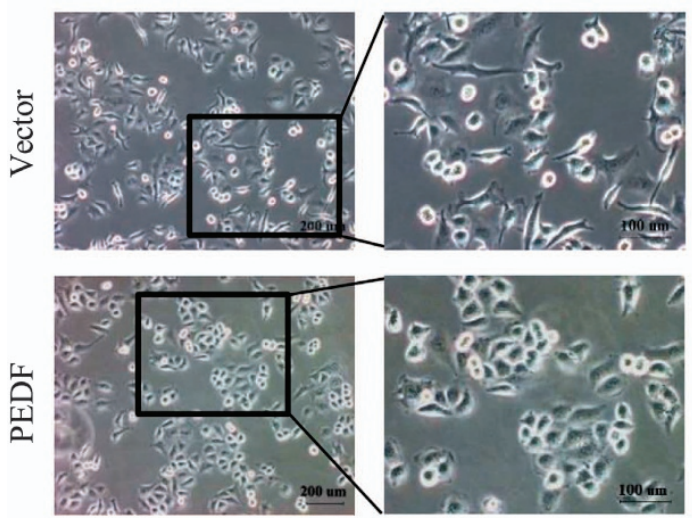

b Vector
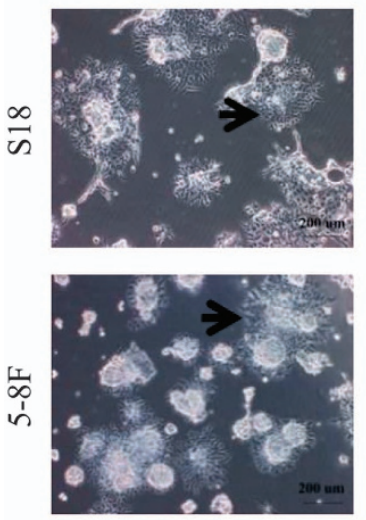

d

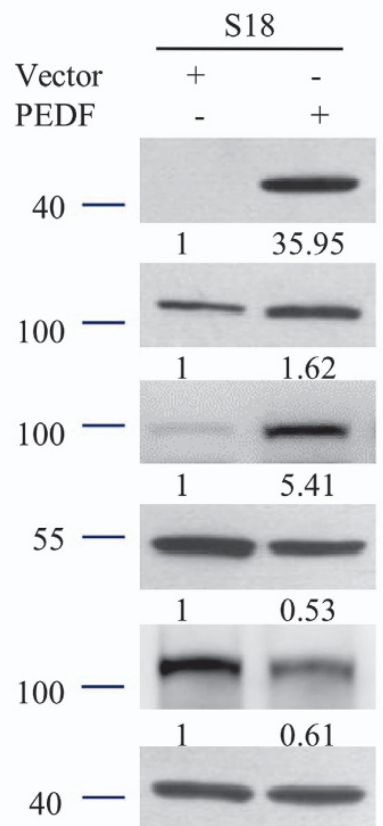

PEDF
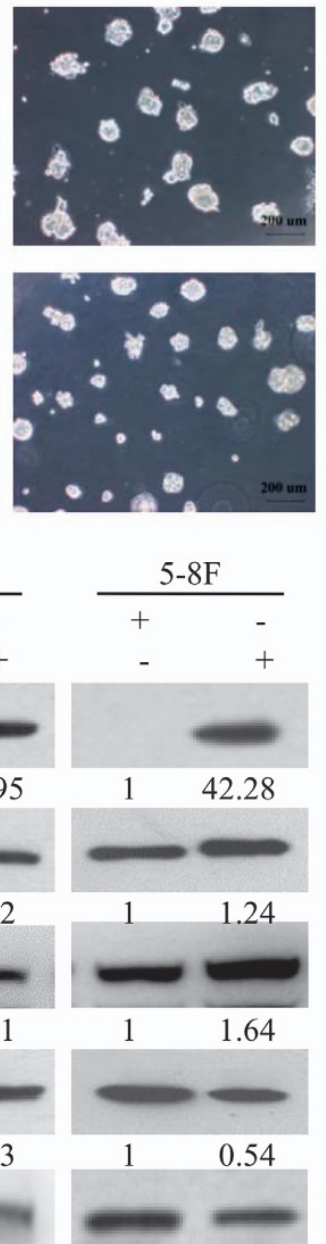

0.64

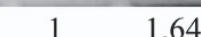

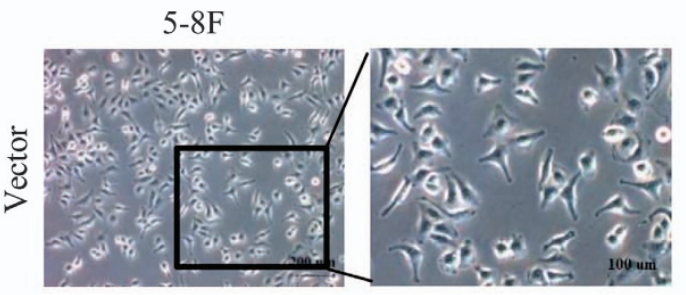

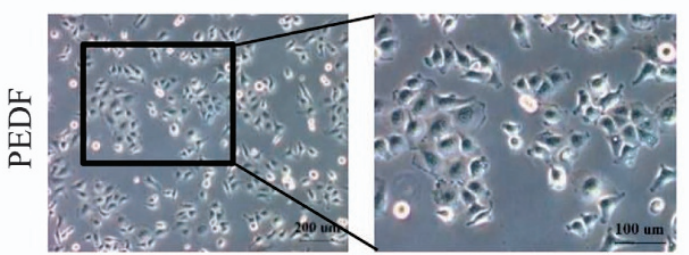

c
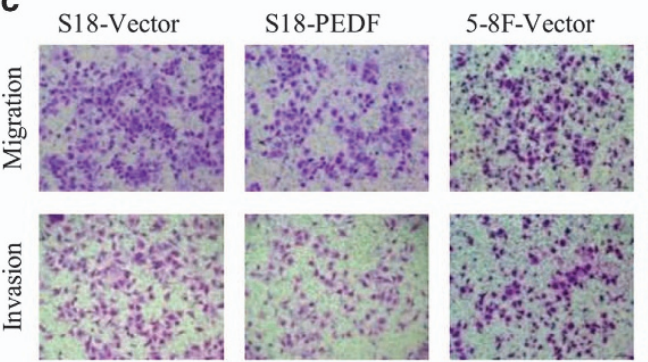

5-8F-PEDF
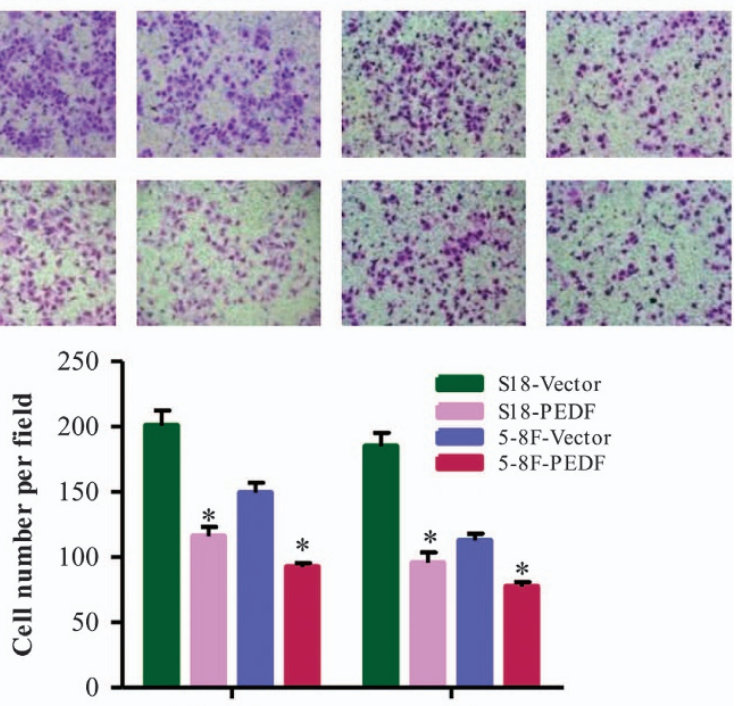

PEDF

Migration

Invasion

\section{E-cadherin}

$\alpha$-catenin

Vimentin

$\mathrm{N}$-cadherin

$\beta$-actin

Figure 3 Overexpression of PEDF induces restoration of the epithelial phenotype. (a) Cell morphology was evaluated by phase-contrast microscopy. (b) Representative micrographs of indicated cells grown on Matrigel for 8 days in 3D spheroid invasion assay. (c) The migration/invasion ability of indicated cells was evaluated by Transwell assay. (d) Expression of epithelial cell markers (E-cadherin, $\alpha$-catenin) and mesenchymal cell markers (Vimentin, $\mathrm{N}$-cadherin) in indicated cells were examined by WB. $\beta$-Actin was used as a loading control. Bars correspond to mean \pm standard deviation (S.D.), ${ }^{*} P<0.05$ 
a

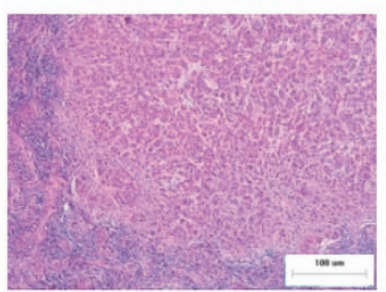

CNE-2-shPEDF

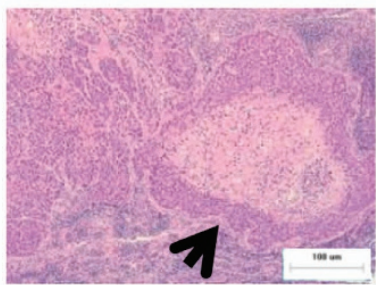

S18-Vector

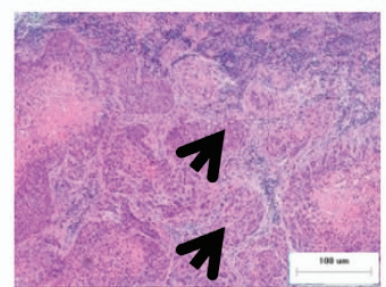

S18-PEDF

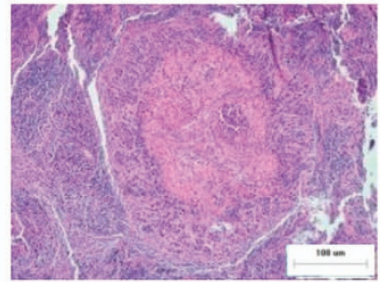

b
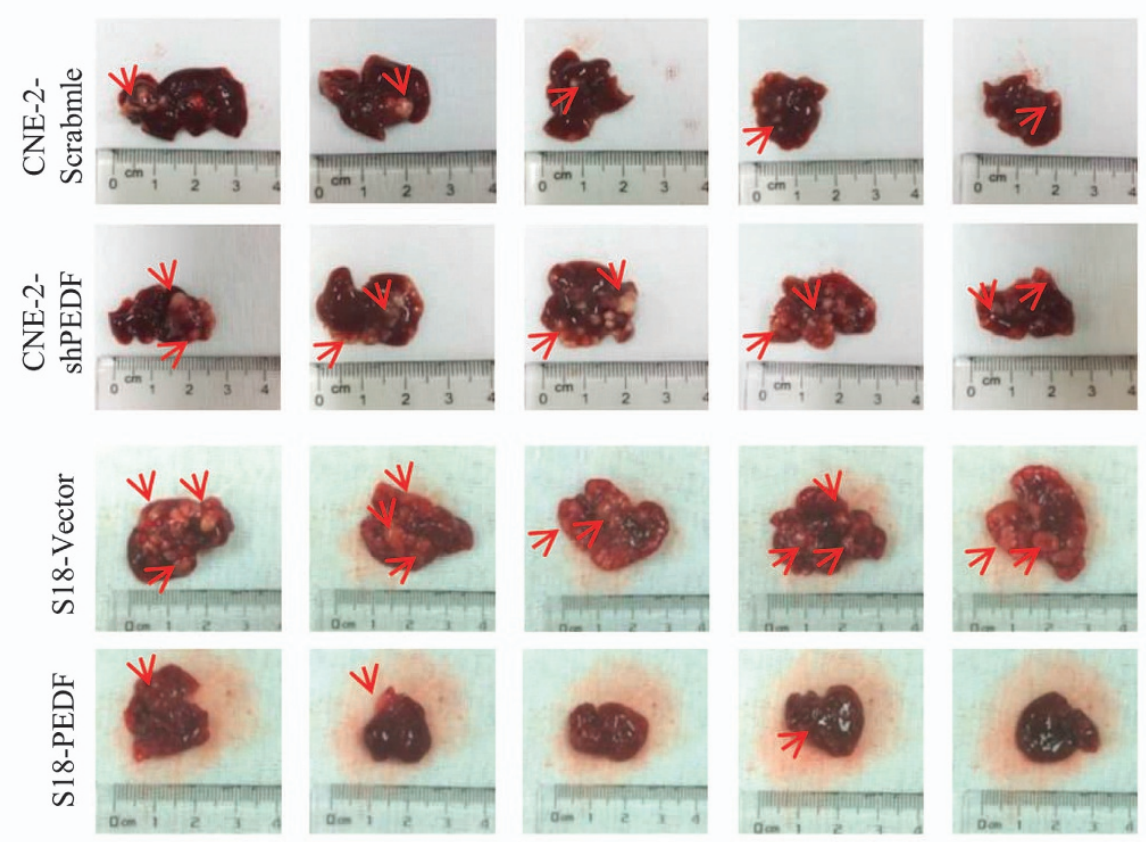

C

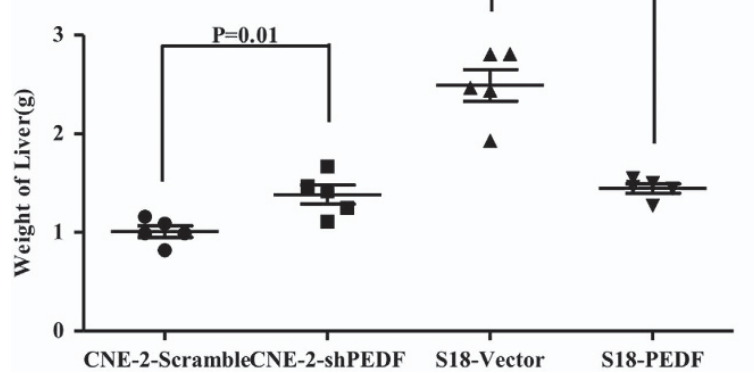

$P=0.0003 \quad$ d

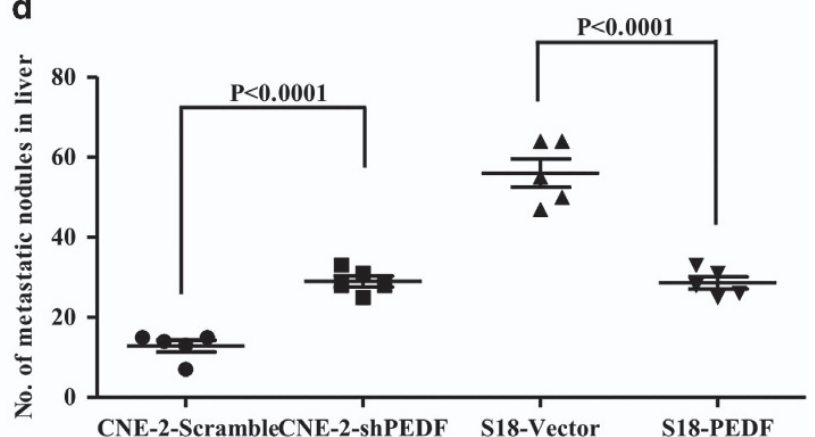

Figure 4 PEDF inhibits the spontaneous liver metastasis of NPC cells in vivo. (a) Representative H\&E staining of indicated spleen orthotopic tumors was shown. Arrows indicate the small metastases. (b) Representative bright-field image of the livers was shown. Arrows indicate surface metastatic nodules. (c and d) Liver weights and metastatic modules number in nude mice that received transplants of indicated cells. Bars correspond to mean \pm standard deviation (S.D.), ${ }^{*} P<0.05$

Cellular signaling pathways, including the Wnt/ $\beta$-catenin, ${ }^{33-35}$ $\mathrm{NF}-\kappa \mathrm{B}^{36,37}$ and $\mathrm{PI} \mathrm{KK} / \mathrm{Akt}^{6,38}$ pathways have been found to be aberrantly activated and have vital roles in the development and progression of NPC. In this study, we observed increased phosphorylation of GSK3 $\beta$ and decreased snail in PEDFoverexpressed NPC cells (Supplementary Figure 3A). Furthermore, the restored epithelial marker and decreased cellular motility triggered by PEDF overexpression were eliminated by GSK3 $\beta$ inhibitor CHIR99021 (Supplementary Figures 3B and $\mathrm{C})$. Phosphorylation of GSK3 $\beta$ could be regulated by PI3K/Akt, MAPK and Wnt/ $\beta$-catenin. ${ }^{39}$ PEDF decreased $\beta$-catenin and nuclear translocation in high-metastatic S18 and 5-8 F cells (Figures $5 \mathrm{a}$ and b). However, no phosphorylation changes of Akt and MAPK were observed in the PEDF-treated S18 and 5-8 F cells (Supplementary Figure 3A). Thus, the GSK3 $\beta$-mediated $\mathrm{Wnt} / \beta$-catenin pathway rather than Akt pathway seems to have a critical role in PEDF-inhibited EMT.

It is believed that the diminished PEDF expression in various tumor types may in part account for increased malignant characteristics during tumor progression. ${ }^{40}$ Possible regulatory mechanisms underlying the changes in PEDF expression during transforming and malignant progression of cancer 

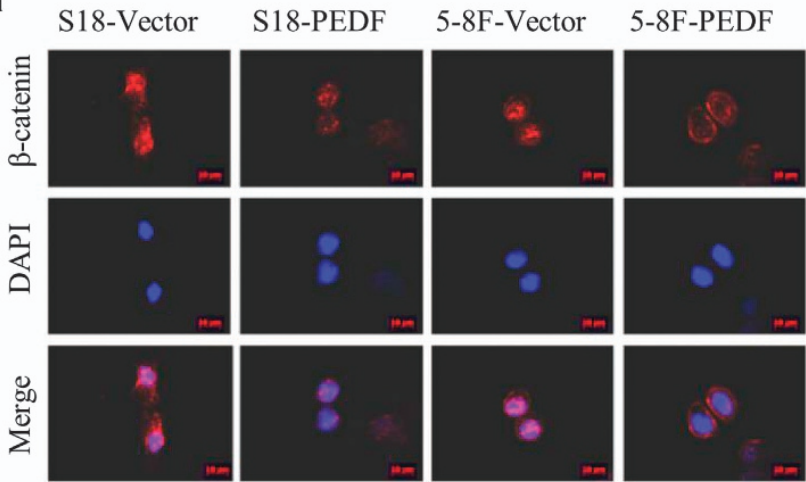

c

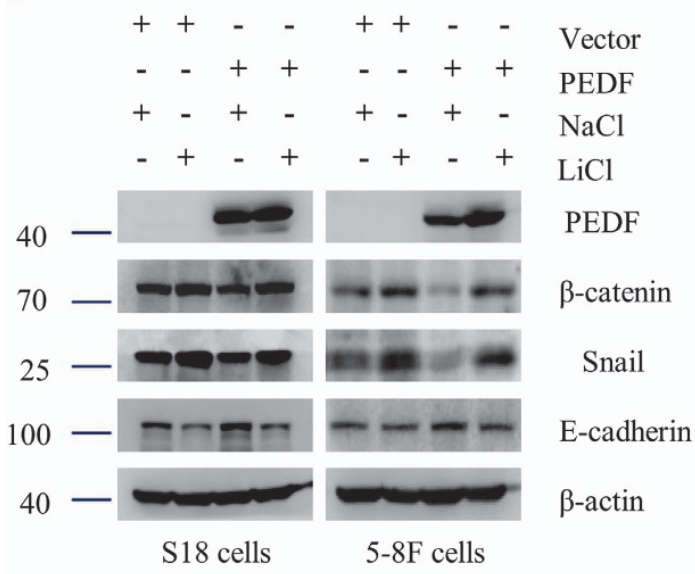

e

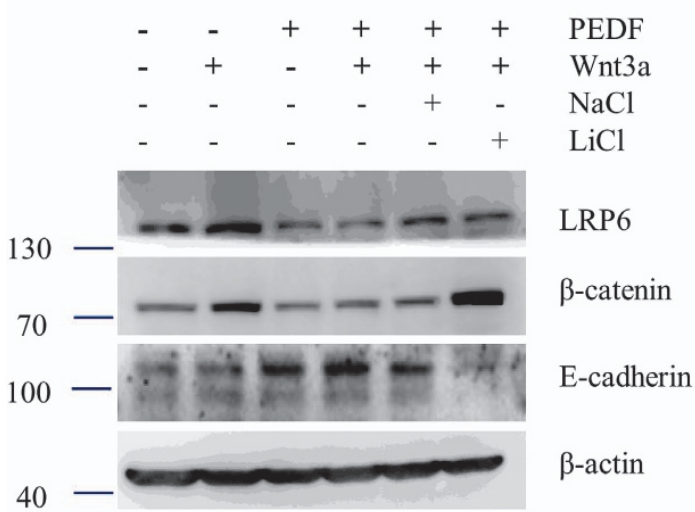

b

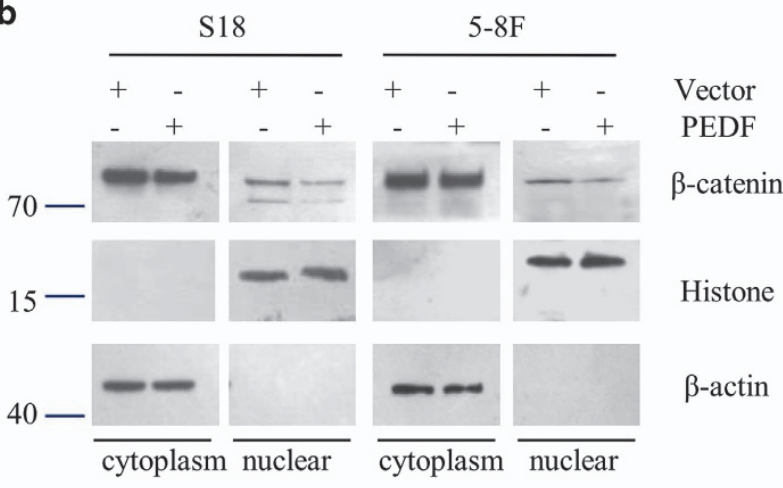

d

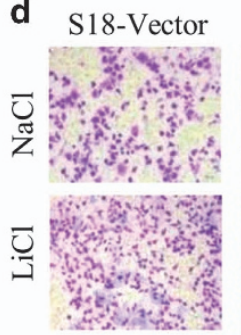

S18-PEDF

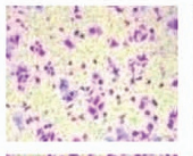

$5-8 \mathrm{~F}-$ Vector

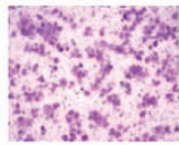

5-8F-PEDF

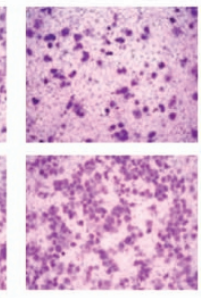

- $\mathrm{NaCl}$
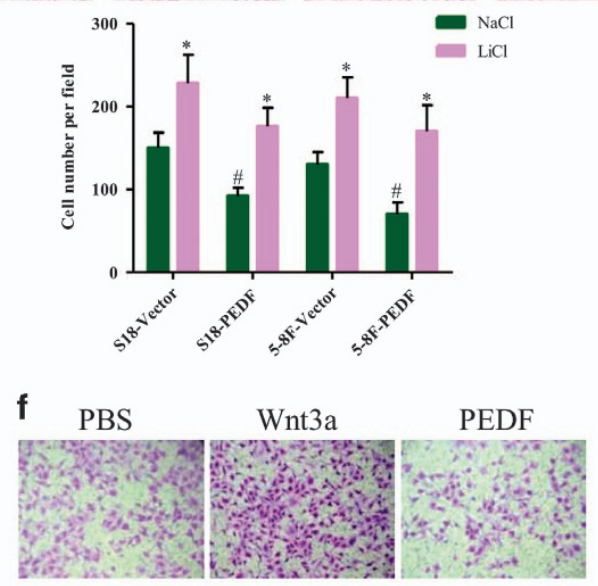

$\mathrm{PEDF}+\mathrm{Wnt} 3 \mathrm{a} \quad \mathrm{P}+\mathrm{W}+\mathrm{NaCl} \quad \mathrm{P}+\mathrm{W}+\mathrm{LiCl}$
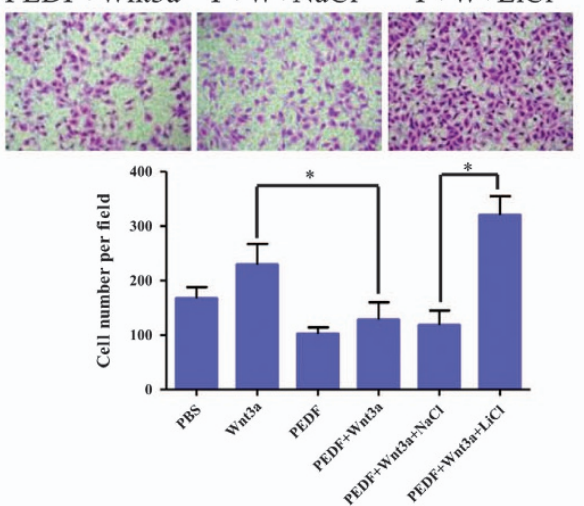

Figure $5 \mathrm{Wnt} / \beta$-catenin signaling pathway is required for PEDF-mediated EMT. (a) Subcellular $\beta$-catenin localization in indicated cells was assessed by immunofluorescence staining. (b) Nuclear fractions of indicated cells were analyzed by WB analysis. (c and d) Cells were treated with Wnt/ $\beta$-catenin signaling agonist LiCl (50 nM) for $16 \mathrm{~h}$, and then performed WB assay or subjected to Transwell/migration assay. ${ }^{*} P<0.05$, relative to $\mathrm{NaCl}$ treatment of the same cell type as controls, ${ }^{\#} P<0.05$, relative to that of Vector with the same treatment as controls. (e and f) Cells were treated with $100 \mathrm{ng} / \mathrm{ml} \mathrm{Wnt3a}$ in the absence or presence of PEDF for $48 \mathrm{~h}$, and then performed WB assay or subjected to Transwell/migration assay. Bars correspond to mean \pm standard deviation (S.D.), ${ }^{\star} P<0.05$ 


\section{a}

$\begin{array}{cccc}\text { PEDF-CDS } & 5^{`}{ }_{481} \text { GGCCCUCUCGGCCCUCUCGCUG }{ }_{502} 3^{\prime} \\ & & |||||||||||| & || \mid \\ \text { miR-320c } & 3^{\prime} & \text { UGGGAGAGUUGGGU } & \text { CGAAAA 5' }\end{array}$

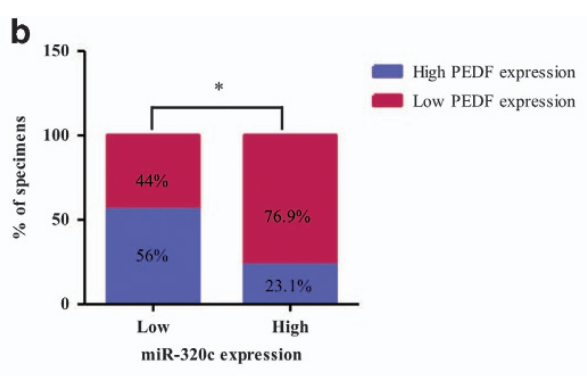

C

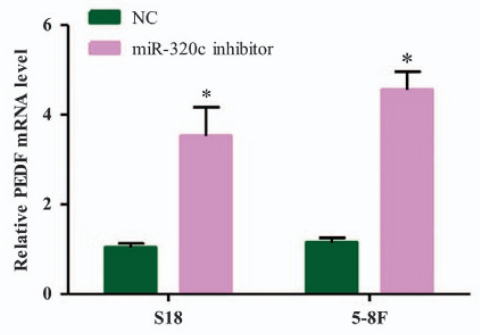

d

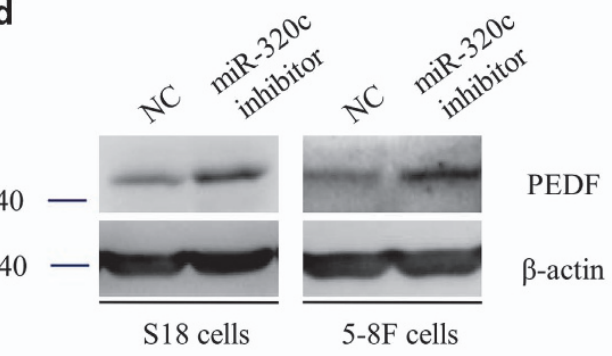

e

PEDF CDS wt TGTCTCCTCTCAGTGTGGCCACGGCCCTCTCGGCCCTCTCGCTGGGAGCGGAGCAGCGAACAG PEDF CDS mut TGTCTCCTCTCAGTGTGGCCACGCAGTGCCATCGAGTCTCGCTGGGAGCGGAGCAGCGAACAG
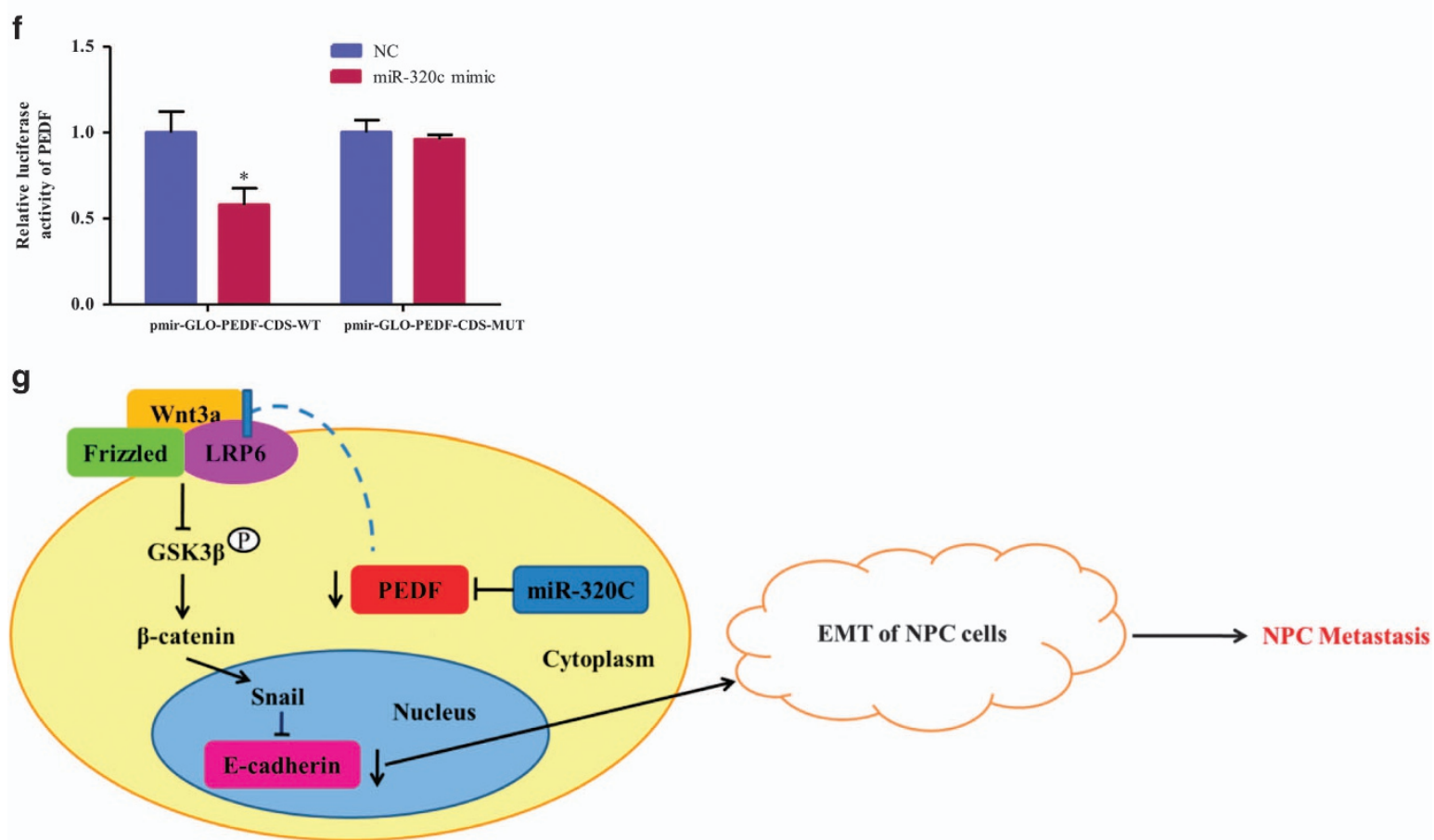

Figure 6 PEDF is a direct target of miR-320c. (a) Schematic miR-320c putative target sites in the CDS of PEDF. (b) Percentage of specimens showing low or high miR-320c expression with the expression levels of PEDF. (c and d) Cells were transfected with miR-320c inhibitors for $48 \mathrm{~h}$, and then performed WB or qRT-PCR analysis. (e) The sequence of PEDF-mut. (f) Indicated plasmids were co-transfected with NC or miR-320c mimics, respectively, for $48 \mathrm{~h}$ and then performed luciferase reporter assay, pmir-GLO was transfected as the internal control. (g) The schematic overview of the potential mechanism involved in PEDF-mediated EMT in NPC. Bars correspond to mean \pm standard deviation (S.D.), ${ }^{*} P<0.05$

are rarely studied, which include transcriptional regulation, hypoxia-mediated regulation and posttranslational modifications. ${ }^{41}$ Our findings suggested that PEDF is suppressed by miRNAs in NPC, indicative of a novel mechanism of PEDF regulation (Figure 6). To assess the causative role of
miR-320c in the loss of PEDF, miR-320c inhibitors were infected into high-metastatic $S 18$ and $5-8 \mathrm{~F}$ cells. The results showed both of $\beta$-catenin and EMT are inhibited by miR-320c inhibitors (Supplementary Figures $4 \mathrm{~A}$ and B). Furthermore, pathological tissue analyses demonstrated that miR-320 was 
increased in many types of cancer, including breast cancer, retinoblastoma and neuroblastoma, while PEDF is less in this kind of cancer types, ${ }^{42-44}$ which was similar to our findings. Moreover, previous studies also revealed that miR-320 with the pro-angiogenic function is decreased in type 2 diabetes. ${ }^{45,46}$ These results indicate that PEDF may be regulated by miR-320c in different types of diseases. Interestingly, in this study, PEDF was regulated by miR-320c at its CDS site rather than $3^{\prime}$-UTR (Supplementary Figures $5 A-C$ ). The detail points for the regulation of miR-320c on PEDF in CDS site needs to be explored in the future.

PEDF has been found to have prognostic value in various types of malignancies, including colorectal cancer, pancreatic cancer, lung cancer and breast cancer. ${ }^{14-17}$ In this study, we observed PEDF have prognostic value in 42 months survival of NPC patients. However, we failed to identify a significant relation between PEDF and patient outcomes in NPC patients' 5 years survival (Supplementary Table 4). This may be due to only $7.3 \%$ (16/218) PEDF-positive expression (Figure 1b) and the 5 years survival of NPC patients is very high (158/218, $72.4 \%$ ). We believe it is important to perform another clinical study with a larger cohort of PEDF-positive specimens and longer follow-up time to further clarify the correlation between PEDF expression and patient outcomes. But, even so, our data demonstrated that the expression pattern of PEDF was remarkably different between NET and NPC, which indicates that PEDF could be as a clinical adjuvant diagnosis marker of NPC.

In summary, PEDF is markedly decreased in NPC tissues and correlated with clinicopathological features. Deficiency of PEDF in nasopharyngeal carcinoma cells triggers the EMT and metastasis in vitro and in vivo via activation of $\mathrm{Wnt} / \beta$ catenin pathway. The downregulation of PEDF was regulated by miR-320c directly in NPC cells. These findings have raised a new mechanism for the EMT and metastasis of NPC, proposed a new function of PEDF and suggested PEDF serving as a potential diagnosis, treatment and prognosis candidate for nasopharyngeal carcinoma.

\section{Materials and Methods \\ Clinical samples. The tissue samples of nasopharyngeal cancer (NPC; $n=218$ ) or nasopharynx epithelial tissues (NET; $n=12$ ) used in Figures 1a and $b$ and Table 1 were provided by the Department of Molecular Diagnostics (Sun Yat- sen University Cancer Center, Guangzhou, China). For Figures 1c-e and Figure 6b, paraffin-embedded tissues from a total of 12 normal controls and 20 NPC tissues, and fresh frozen samples of NET $(n=11)$ or NPC $(n=27)$ were provided by the cancer center (Affiliated Hospital of Guangdong Medical College, Zhanjiang, China). None of the patients had received any chemotherapy or radiotherapy before their operation. Data of clinicopathological parameters were obtained from patients clinical records and pathological reports. This study conforms to the principles outlined in the Declaration of Helsinki, approved by the Medical Ethics Committee of Sun Yat-Sen University and Guangdong Medical College. Written informed consents were obtained from the donors.}

Immunohistochemical staining. Paraffin-embedded tissues were sectioned at $5 \mu \mathrm{m}$ and immunohistochemistry assays were performed. Briefly, the sections were hydrated, blocked and incubated with a series of antibodies overnight at $4{ }^{\circ} \mathrm{C}$. The Dako real EnVision kit (Dako, Santa Clara, CA, USA) was used to detect the primary antibodies followed by 3, 3-diaminobenzidine substrate visualization and counterstaining with hematoxylin. The results were evaluated by assessing staining intensity according to the method described by Remmele and Stegner. ${ }^{47}$ The average value from two referees was used as the final score.
Cell culture. The NPC cell lines CNE-2 and its subclones $S 18$ were provided by Professor Qian Chao-nan. ${ }^{38}$ The SUNE-1 and its subclones 5-8 F were the kind gifts of Professor Zeng Mu-sheng at Sun Yat-sen University Cancer Center. ${ }^{10}$ All cell lines were cultured for less than 50 passages. The NPC cell lines were maintained in RPIM 1640 medium (Hyclone, Beijing, China) supplemented with 10\% fetal bovine serum (Gibco, Montevideo, Uruguay). All the cell lines were incubated at $37^{\circ} \mathrm{C}$ in a $5 \% \mathrm{CO}_{2}$ incubator. For $3 \mathrm{D}$ cell culture, $1 \times 10^{4}$ cells were seeded on Matrigel-coated 24-well plates and the complete medium containing $2 \%$ Matrigel was refreshed every day. The cells forming spheres were photographed at 2-day intervals for 8 days.

RNA extraction and qRT-PCR. Small RNA was extracted from samples using RNAiso Kit for Small RNA (TaKaRa, Dalian, China) and subsequently reverse transcribed into cDNA by One Step PrimeScript miRNA cDNA Synthesis Kit (TaKaRa). Meanwhile, total RNA from samples was extracted using Ultrapure RNA Kit (KangWei, Beijing, China) and transcribed into cDNA using PrimeScript RT reagent Kit (TaKaRa). The sequences of PCR primers were shown in Supplementary Table 1. The quantitative PCR was performed using SYBR Premix Ex Taq (TaKaRa) and a LightCycler 2.0 Real Time PCR system (Roche Diagnostics, Rotkreuz, Switzerland). U6 and $\beta$-actin were used for normalizing the expression of miRNA and mRNA, respectively. The experiments were performed in triplicate.

Lentiviral transduction studies. Cell lines stably expressing PEDF were established using a ViraPower Lentiviral Packaging Kit (Invitrogen, Carlsbad, CA, USA) according to the manufacturer's instructions. Briefly, oligos containing the sequences of PEDF were cloned into a lentiviral vector. The lentiviral particles were prepared by transient co-transfection of the resulting vector and ViraPower Lentiviral Packaging Mix into 293FT cells using Lipofectamine 2000 (Invitrogen). Infectious lentiviruses were harvested $72 \mathrm{~h}$ after transfection, centrifuged to remove cell debris, filtered through $0.45 \mu \mathrm{m}$ filter (Millipore, Bedford, MA, USA) and concentrated by centrifugal filter device (Millipore). Lentiviruses expressing either PEDF short hairpin RNA (shPEDF) or a Scrambled nontarget shRNA were established by Genechem company. The target for human PEDF shRNA was 5'-GGATCGTCTTTGAGAAGAA-3'. The NPC cells were transduced with lentiviruses. After 7 days, $1 \mu \mathrm{g} / \mathrm{ml}$ puromycin (Sigma-Aldrich, St Louis, MO, USA) was added to the culture and lasted for 10 days. Western blot and qRT-PCR were performed to analyze the PEDF expression.

Western blot. The cells were washed with cold PBS and collected using the RIPA lysis buffer (Santa Cruz, St, Dallas, TX, USA). Nuclear extracts were obtained using the NE-PER Nuclear and cytoplasmic extraction reagents (Thermo, Rockford, AL, USA) according to the manufacturer's instructions. The concentration of total protein in the lysate was measured using the Bio-Rad protein assay kit (Bio-Rad, Hercules, CA, USA). An equal amount of protein in each sample was loaded onto $12 \%$ SDS-PAGE gels and transferred to PVDF membranes (Roche Diagnostics). The membrane was probed with the first antibody listed in Supplementary Table 2 at $4{ }^{\circ} \mathrm{C}$ overnight and then with the peroxidase-conjugated secondary antibody, detected with ECL kit (APPLYGEN, Beijing, China). The same membrane was stripped and incubated with an anti- $\beta$-actin antibody for normalization.

Migration and invasion assays. A total of $2 \times 10^{4}$ (for S18 and 5-8 F) or $4 \times 10^{4}$ (for CNE-2 and SUNE-1) cells in $200 \mu \mathrm{l}$ serum-free RPIM 1640 were seeded on cell culture inserts with $8 \mu \mathrm{m}$ microporous filters (Corning, Corning, NY, USA) coated with (invasion) or without (migration) Matrigel (BD Biosciences, Franklin Lakes, NJ, USA), and $800 \mu \mathrm{l}$ of RPIM 1640 containing 10\% FBS was added to the lower chamber. After being incubated for indicated times, the cells in the upper filters (inside the inserts) were removed, and the migrated or invaded cells in the lower filters (outside the inserts) were fixed in ethanol for $20 \mathrm{~min}$, then stained with crystal violet for $10 \mathrm{~min}$ and counted under a microscope. The number of migrated or invaded cells in five random optical fields (x100 magnification) of each filter from triplicate inserts was averaged. Experiments were performed in triplicate.

Immunofluorescence analysis. The cells were seeded on coverslips and incubated for $24 \mathrm{~h}$, washed with cold PBS three times and fixed with ice-cold paraformaldehyde for $10 \mathrm{~min}$ at $-20^{\circ} \mathrm{C}$. Next, the cells were permeabilized for 10 min with $0.01 \%$ Triton X-100 in PBS. Then the cells were blocked for 30 min in normal goat serum and incubated with the anti- $\beta$-catenin antibody in antibody diluents (Dako) overnight at $4^{\circ} \mathrm{C}$. After washing with PBS three times, the slides were incubated for $1 \mathrm{~h}$ in the dark at $37^{\circ} \mathrm{C}$ with the Rhodamine-conjugated 
secondary antibody (Sigma-Aldrich). After three further washes, the slides were stained with 4',6-diamidino- 2-phenylindole (DAPI, Sigma-Aldrich) for $10 \mathrm{~min}$ to counterstain the nucleus, and examined using the Axio Imager Z1 microscope (ZEISS, Oberkochen, Baden-Wuerttemberg, Germany). In negative control staining, the primary antibodies were omitted.

Animals and liver metastasis assay. BALB/c-nu male mice (16-18 g), at age 5-6 weeks, were purchased from the Laboratory Animal Center of Guangdong Province (Guangzhou, China) and fed in a specific pathogen-free environment. The mice were randomly grouped into two groups (five mice/group). The NPC cells $\left(2 \times 10^{5}\right.$ in $30 \mu$ l of $33 \%$ Matrigel) were injected into the spleens of laparotomized nude mice using insulin syringes (BD Biosciences) as previously reported. ${ }^{38}$ After 28 days, the experiment was terminated. The liver and spleen of each mouse were weighed and the metastatic nodules in each liver were counted. The sections from paraffin-embedded samples were performed H\&E staining for histological evidence of the tumor phenotype. The care, use and treatment of all animals were in strict agreement with the institutionally approved protocol according to the USPHS Guide for the care and use of laboratory animals, as well as the guidelines outlined in Care and Use of Laboratory Animals by the Sun Yat-sen University in this study.

Luciferase reporter assay. A total $1 \times 10^{4}$ cells were seeded in triplicate in 96-well plates. Indicated plasmids plus NC or miR-320c mimics were transfected into the 293 A cells using Lipofectamine 2000 (Invitrogen). Forty-eight hours post transfection, Dual-Glo Luciferase Assay System (Promega, Madison, SD, USA) was performed according to the manufacturers' instruction. Renilla luciferase assay was used to normalize the corresponding firefly luciferase activity.

Statistical analysis. Differences among variables were assessed by two-tailed Student's unpaired $t$-tests. The categorical data were analyzed by the Chi-square method. Data were presented as the mean \pm standard deviation (S.D.). $P<0.05$ was considered statistically significant. The statistical analyses were performed using SPSS 18.0 (Chicago, IL, USA) or GraphPad Prism 5.0.

\section{Conflict of Interest}

The authors declare no conflict of interest.

Acknowledgements. This study was supported by National Natural Science Foundation of China $(81272338,81471033,81272515,81572342$ and 81570871), National Key Sci-Tech Special Project of China (2013ZX09102-053 and 2015GKS-355), Key Project of Nature Science Foundation of Guangdong Province, China (2015A030311043 and 2016A030311035), Guangdong Natural Science Fund (2014A030313073 and 2015A030313103), Guangdong Science and Technology Project (2015B090903063), Key Sci-tech Research Project of Guangzhou Municipality, China (201508020033), The Ministry of Education of the People's Republic of China (985 project PCSIRT 0947, 13ykpy06, 14ykpy05 and 20130171110053), Guangzhou Science Technology and Innovation Commission (201610010186). We thank Qian Chao-nan and Zeng Mu-sheng for providing the NPC cell lines. The funding agencies had no role in the study design, data collection and analysis, decision to publish or preparation of the manuscript.

1. Yu MC, Yuan JM. Epidemiology of nasopharyngeal carcinoma. Semin Cancer Biol 2002; 12 : 421-429.

2. Lee AW, Lau WH, Tung SY, Chua DT, Chappell R, Xu L et al. Preliminary results of a randomized study on therapeutic gain by concurrent chemotherapy for regionally-advanced nasopharyngeal carcinoma: NPC-9901 Trial by the Hong Kong Nasopharyngeal Cancer Study Group. J Clin Oncol 2005; 23: 6966-6975.

3. Kalluri R, Weinberg RA. The basics of epithelial-mesenchymal transition. J Clin Invest 2009; 119: $1420-1428$.

4. Wang L, Tian WD, Xu X, Nie B, Lu J, Liu X et al. Epstein-Barr virus nuclear antigen 1 (EBNA1) protein induction of epithelial-mesenchymal transition in nasopharyngeal carcinoma cells. Cancer 2014; 120: 363-372.

5. Horikawa T, Yoshizaki T, Kondo S, Furukawa M, Kaizaki Y, Pagano JS. Epstein-Barr Virus latent membrane protein 1 induces Snail and epithelial-mesenchymal transition in metastatic nasopharyngeal carcinoma. Br J Cancer 2011; 104: 1160-1167.

6. Kong QL, Hu LJ, Cao JY, Huang YJ, Xu LH, Liang Y et al. Epstein-Barr virus-encoded LMP2A induces an epithelial-mesenchymal transition and increases the number of side population stem-like cancer cells in nasopharyngeal carcinoma. PLoS Pathog 2010; 6: e1000940.
7. Horikawa T, Yang J, Kondo S, Yoshizaki T, Joab I, Furukawa M et al. Twist and epithelialmesenchymal transition are induced by the EBV oncoprotein latent membrane protein 1 and are associated with metastatic nasopharyngeal carcinoma. Cancer Res 2007; 67: 1970-1978.

8. Nelson WJ, Nusse R. Convergence of Wnt, beta-catenin, and cadherin pathways. Science 2004; 303: 1483-1487.

9. Zeng ZY, Zhou YH, Zhang WL, Xiong W, Fan SQ, Li XL et al. Gene expression profiling of nasopharyngeal carcinoma reveals the abnormally regulated Wnt signaling pathway. Hum Pathol 2007; 38: 120-133.

10. Zong D, Yin L, Zhong Q, Guo WJ, Xu JH, Jiang $N$ et al. ZNF 488 enhances the invasion and tumorigenesis in nasopharyngeal carcinoma via the Wnt signaling pathway involving epithelial mesenchymal transition. Cancer Res Treat 2016; 48: 334-344.

11. Ren $X Y$, Zhou GQ, Jiang W, Sun Y, Xu YF, Li YQ et al. Low SFRP1 expression correlates with poor prognosis and promotes cell invasion by activating the Wnt/beta-catenin signaling pathway in NPC. Cancer Prev Res (Phila) 2015; 8: 968-977.

12. Qin L, Yin YT, Zheng FJ, Peng LX, Yang CF, Bao YN et al. WNT5A promotes stemness characteristics in nasopharyngeal carcinoma cells leading to metastasis and tumorigenesis. Oncotarget 2015; 6: 10239-10252.

13. Dawson DW, Volpert OV, Gillis $\mathrm{P}$, Crawford SE, Xu H, Benedict W et al. Pigment epitheliumderived factor: a potent inhibitor of angiogenesis. Science 1999; 285: 245-248.

14. Cai J, Parr C, Watkins G, Jiang WG, Boulton M. Decreased pigment epithelium-derived factor expression in human breast cancer progression. Clin Cancer Res 2006; 12: 3510-3517.

15. Zhang L, Chen J, Ke Y, Mansel RE, Jiang WG. Expression of pigment epithelial derived factor is reduced in non-small cell lung cancer and is linked to clinical outcome. Int J Mol Med 2006; 17: 937-944.

16. Uehara $\mathrm{H}$, Miyamoto $\mathrm{M}$, Kato $\mathrm{K}$, Ebihara $\mathrm{Y}$, Kaneko $\mathrm{H}$, Hashimoto $\mathrm{H}$ et al. Expression of pigment epithelium-derived factor decreases liver metastasis and correlates with favorable prognosis for patients with ductal pancreatic adenocarcinoma. Cancer Res 2004; 64 3533-3537.

17. Ji D, Li M, Zhan T, Yao Y, Shen J, Tian $\mathrm{H}$ et al. Prognostic role of serum AZGP1, PEDF and PRDX2 in colorectal cancer patients. Carcinogenesis 2013; 34: 1265-1272.

18. Lamouille S, Xu J, Derynck R. Molecular mechanisms of epithelial-mesenchymal transition Nat Rev Mol Cell Biol 2014; 15: 178-196.

19. Park K, Lee K, Zhang B, Zhou T, He X, Gao G et al. Identification of a novel inhibitor of the canonical Wnt pathway. Mol Cell Biol 2011; 31: 3038-3051.

20. Qi W, Yang C, Dai Z, Che D, Feng J, Mao Y et al. High levels of pigment epithelium-derived factor in diabetes impair wound healing through suppression of Wnt signaling. Diabetes 2015; 64: 1407-1419.

21. Wang HY, Yan LX, Shao Q, Fu S, Zhang ZC, Ye W et al. Profiling plasma microRNA in nasopharyngeal carcinoma with deep sequencing. Clin Chem 2014; 60: 773-782.

22. Rehmsmeier M. Fast and effective prediction of microRNA/target duplexes. RNA 2004; 10 : 1507-1517.

23. Faivre S, Janot F, Armand JP. Optimal management of nasopharyngeal carcinoma. Curr Opin Oncol 2004; 16: 231-235.

24. Xu Z, Fang S, Zuo Y, Zhang Y, Cheng R, Wang $Q$ et al. Combination of pigment epitheliumderived factor with radiotherapy enhances the antitumor effects on nasopharyngeal carcinoma by downregulating vascular endothelial growth factor expression and angiogenesis. Cancer Sci 2011; 102: 1789-1798.

25. Song LB, Li J, Liao WT, Feng Y, Yu CP, Hu LJ et al. The polycomb group protein Bmi-1 represses the tumor suppressor PTEN and induces epithelial-mesenchymal transition in human nasopharyngeal epithelial cells. J Clin Invest 2009; 119: 3626-3636.

26. Becerra SP, Fariss RN, Wu YQ, Montuenga LM, Wong P, Pfeffer BA. Pigment epitheliumderived factor in the monkey retinal pigment epithelium and interphotoreceptor matrix: apical secretion and distribution. Exp Eye Res 2004; 78: 223-234.

27. Sonoda S, Sreekumar PG, Kase S, Spee C, Ryan SJ, Kannan R et al. Attainment of polarity promotes growth factor secretion by retinal pigment epithelial cells: relevance to age-related macular degeneration. Aging (Albany NY) 2010; 2: 28-42.

28. Orgaz JL, Ladhani O, Hoek KS, Fernandez-Barral A, Mihic D, Aguilera $O$ et al. 'Loss of pigment epithelium-derived factor enables migration, invasion and metastatic spread of human melanoma'. Oncogene 2009; 28: 4147-4161.

29. Dong BB, Yan JS, Yan YY, Xie TC, Xu L, Hu GH et al. Downregulation of Pigment epitheliumderived factor is associated with increased epithelial-mesenchymal transition (EMT) in bladder cancer. Panminerva Med 2016; 59: 9-14.

30. Zhou D, Zhang M, Xu P, Yu Y, Ye G, Zhang L et al. Expression of pigment epithelium-derived factor is associated with a good prognosis and is correlated with epithelial-mesenchymal transition-related genes in infiltrating ductal breast carcinoma. Oncol Lett 2016; 11: 116-124.

31. Hong H, Zhou T, Fang S, Jia M, Xu Z, Dai Z et al. Pigment epithelium-derived factor (PEDF) inhibits breast cancer metastasis by down-regulating fibronectin. Breast Cancer Res Treat 2014; 148: 61-72.

32. Waerner T, Alacakaptan M, Tamir I, Oberauer R, Gal A, Brabletz T et al. ILEl: a cytokine essential for EMT, tumor formation, and late events in metastasis in epithelial cells. Cancer Cell 2006; 10: 227-239.

33. Hsu CY, Yi YH, Chang KP, Chang YS, Chen SJ, Chen HC. The Epstein-Barr virus-encoded microRNA MiR-BART9 promotes tumor metastasis by targeting E-cadherin in nasopharyngeal carcinoma. PLoS Pathog 2014; 10: e1003974. 
34. Xia H, Cheung WK, Sze J, Lu G, Jiang S, Yao H et al. miR-200a regulates epithelialmesenchymal to stem-like transition via ZEB2 and beta-catenin signaling. J Biol Chem 2010; 285: 36995-37004.

35. Song Y, Yang QX, Zhang F, Meng F, Li H, Dong Y et al. Suppression of nasopharyngeal carcinoma cell by targeting beta-catenin signaling pathway. Cancer Epidemiol 2012; 36: e116-e121.

36. Valentine R, Dawson CW, Hu C, Shah KM, Owen TJ, Date KL et al. Epstein-Barr virus-encoded EBNA1 inhibits the canonical NF-kappaB pathway in carcinoma cells by inhibiting IKK phosphorylation. Mol Cancer 2010; 9: 1.

37. Cheung AK, Ko JM, Lung HL, Chan KW, Stanbridge EJ, Zabarovsky E et al. Cysteine-rich intestinal protein 2 (CRIP2) acts as a repressor of NF-kappaB-mediated proangiogenic cytokine transcription to suppress tumorigenesis and angiogenesis. Proc Natl Acad Sci USA 2011; 108: 8390-8395.

38. Li XJ, Peng LX, Shao JY, Lu WH, Zhang JX, Chen S et al. As an independent unfavorable prognostic factor, IL-8 promotes metastasis of nasopharyngeal carcinoma through induction of epithelial-mesenchymal transition and activation of AKT signaling. Carcinogenesis 2012; 33: 1302-1309.

39. Zhou BP, Deng J, Xia W, Xu J, Li YM, Gunduz M et al. Dual regulation of Snail by GSK-3beta-mediated phosphorylation in control of epithelial-mesenchymal transition. Nat Cell Biol 2004; 6: 931-940.

40. Belkacemi L, Zhang SX. Anti-tumor effects of pigment epithelium-derived factor (PEDF) implication for cancer therapy. A mini-review. J Exp Clin Cancer Res 2016; 35: 4.

41. Becerra SP, Notario V. The effects of PEDF on cancer biology: mechanisms of action and therapeutic potential. Nat Rev Cancer 2013; 13: 258-271.

42. Bronisz A, Godlewski J, Wallace JA, Merchant AS, Nowicki MO, Mathsyaraja $\mathrm{H}$ et al Reprogramming of the tumour microenvironment by stromal PTEN-regulated miR-320. Nat Cell Biol 2012; 14: 159-167.

43. Zhao JJ, Yang J, Lin J, Yao N, Zhu Y, Zheng $\mathrm{J}$ et al. Identification of miRNAs associated with tumorigenesis of retinoblastoma by miRNA microarray analysis. Childs Nerv Syst 2009; 25: 13-20.
44. Schulte JH, Horn S, Otto T, Samans B, Heukamp LC, Eilers UC et al. MYCN regulates oncogenic MicroRNAs in neuroblastoma. Int $J$ Cancer 2008; 122: 699-704.

45. Zampetaki A, Kiechl S, Drozdov I, Willeit P, Mayr U, Prokopi M et al. Plasma microRNA profiling reveals loss of endothelial miR-126 and other microRNAs in type 2 diabetes. Circ Res 2010; 107: 810-817.

46. Chiavacci E, Rizzo M, Pitto L, Patella F, Evangelista M, Mariani L et al. The zebrafish/tumor xenograft angiogenesis assay as a tool for screening anti-angiogenic miRNAs. Cytotechnology 2014; 67: 969-975.

47. Remmele W, Stegner HE. Recommendation for uniform definition of an immunoreactive score (IRS) for immunohistochemical estrogen receptor detection (ER-ICA) in breast cancer tissue. Pathologe 1987; 8: 138-140.

Cell Death and Disease is an open-access journal published by Nature Publishing Group. This work is licensed under a Creative Commons Attribution 4.0 International License. The images or other third party material in this article are included in the article's Creative Commons license, unless indicated otherwise in the credit line; if the material is not included under the Creative Commons license, users will need to obtain permission from the license holder to reproduce the material. To view a copy of this license, visit http://creativecommons.org/licenses/by/4.0/

(C) The Author(s) 2017

Supplementary Information accompanies this paper on Cell Death and Disease website (http://www.nature.com/cddis) 